\title{
Stability Analysis of Equilibrium Point of Population Competitive Model
}

\author{
Yun Ouyang ${ }^{a}$, Kexin Yuan ${ }^{b}$, Wu-Sheng Wang ${ }^{\mathrm{c}^{*}}$
}

\author{
School of Mathematics and Statistics, Hechi University, Guangxi, Yizhou 546300, P. R. China \\ ashuxueoyy@126.com, b494720432@qq.com, wang4896@126.com
}

Keywords: Two species competitive model; Equilibrium points; Stability; Matlab program; Graphics of phase trajectory.

\begin{abstract}
In this paper, the stability of equilibrium points of two species competitive model are studied. On the base of logistic model, the competitive model of two species living in the same natural environment is considered. Firstly, the four equilibrium points of the two species competition model are given, and then stability of each equilibrium point is judged by ordinary differential equation stability theory. Secondly, according to the characteristics of the model, the phase plane is divided into several regions. Finally, matlab programs are wrote, and the numerical solutions of two species competition model are obtained for several different initial conditions, and the graphics of corresponding phase trajectory are drew, and the stability of equilibrium points are analyzed.
\end{abstract}

\section{Introduction}

Combination of mathematics and ecology provides an effective tool to understand, explain and predict the ecological phenomenon. In the specific ecological environment, generally there are multiple species populations, and the increase or decrease of the quantity of each population is not only affected by their self limiting law, inevitably will be restricted by other populations. In order to compete for the same limited sources of food and living space, there are the mutual competition, and the interdependence or the law of the jungle between two or more than two populations live in the same environment. The result of competition is that the two populations coexist or the weak competitive populations become extinct, while the competitive population can survive and reach the maximum amount of environmental capacity (see [1-10]).

In this paper, we first consider the logistic model of single species, and then consider the model of two competition species living the same natural environment. According to the method for solving equilibrium points of the differential equations, we obtain four equilibrium points of two species competitive model. Then by means of stability theory on the ordinary differential equation, we judge the stability of every equilibrium point. According to the characteristics of the model, we divide the phase plane into several regions. In order to obtain the numerical solution of the two species competitive model under different initial conditions, we write corresponding matlab program, and draw the corresponding to the phase trajectory diagram and analysis of equilibrium stability.

\section{Competitive Model of Two Populations}

When population $\mathrm{A}$ and population $\mathrm{B}$ live alone in a natural environment, the change rate of a population obeys the law of Logistic. Let $y_{1}(t), y_{2}(t)$ denote the number of population $\mathrm{A}$ and population $\mathrm{B}, m_{1}, m_{2}$ their intrinsic growth rate, and $N_{1}, N_{2}$ their maximum capacity. Specifically, the number of populations of A satisfies the following differential equation (see [1])

$$
y_{1}^{\prime}(t)=m_{1} y_{1}\left(1-\frac{y_{1}}{N_{1}}\right)
$$


where $\left(1-\frac{y_{1}}{N_{1}}\right)$ reflect the blocking effect of A on the growth of its own due to the consumption of limited resources, $\frac{y_{1}}{N_{1}}$ can be explained to amount of food which unit number A (be relative to $N_{1}$ ) consume.

When two populations live in the same natural environment, the number of populations of A and B satisfy the following differential equation (see [1])

$$
\left\{\begin{array}{l}
y_{1}^{\prime}(t)=m_{1} y_{1}\left(1-\frac{y_{1}}{N_{1}}-w_{1} \frac{y_{2}}{N_{2}}\right) \\
y_{2}^{\prime}(t)=m_{2} y_{2}\left(1-w_{2} \frac{y_{1}}{N_{2}}-\frac{y_{2}}{N_{2}}\right)
\end{array}\right.
$$

\section{Stability of Equilibrium Points}

According to the method for solving equilibrium points of the differential equations, equilibrium points of model (2) are the solutions of the following algebraic equations

$$
\left\{\begin{array}{l}
f\left(y_{1}, y_{2}\right) \equiv m_{1} y_{1}\left(1-\frac{y_{1}}{N_{1}}-w_{1} \frac{y_{2}}{N_{2}}\right)=0 \\
g\left(y_{1}, y_{2}\right) \equiv m_{2} y_{2}\left(1-w_{2} \frac{y_{1}}{N_{1}}-\frac{y_{2}}{N_{2}}\right)=0
\end{array}\right.
$$

Solving algebraic equations, we can obtain four equilibrium points of model (2) (see [1])

$Q_{1}\left(N_{1}, 0\right), Q_{2}\left(0, N_{2}\right), Q_{3}\left(\frac{N_{1}\left(1-w_{1}\right)}{1-w_{1} w_{2}}, \frac{N_{2}\left(1-w_{2}\right)}{1-w_{1} w_{2}}\right), Q_{4}(0,0)$.

In order to judge the stability of the equilibrium points, Let

$$
A=\left[\begin{array}{ll}
f_{y_{1}} & f_{y_{2}} \\
g_{y_{1}} & g_{y_{2}}
\end{array}\right]=\left[\begin{array}{cc}
m_{1}\left(1-\frac{2 y_{1}}{N_{1}}-\frac{w_{1} y_{2}}{N_{2}}\right) & -\frac{m_{1} w_{1} y_{1}}{N_{2}} \\
-\frac{m_{2} w_{2} y_{2}}{N_{1}} & m_{2}\left(1-\frac{w_{2} y_{1}}{N_{1}}-\frac{2 y_{2}}{N_{2}}\right)
\end{array}\right],
$$

and

$$
\begin{aligned}
& u=-\left.\left(f_{y_{1}}+g_{y_{2}}\right)\right|_{Q_{i}}, i=1,2,3,4, \\
& v=\left.\operatorname{det} A\right|_{Q_{i}}, i=1,2,3,4 .
\end{aligned}
$$

By stability theory on the ordinary differential equation, we know the conditions of stability of four equilibrium points of model (2) are $u>0, v>0$. From $u>0, v>0$, we see that the conditions of stability of three equilibrium points $Q_{1}\left(N_{1}, 0\right), Q_{2}\left(0, N_{2}\right), Q_{3}\left(\frac{N_{1}\left(1-w_{1}\right)}{1-w_{1} w_{2}}, \frac{N_{2}\left(1-w_{2}\right)}{1-w_{1} w_{2}}\right)$ are $w_{1}<1, w_{2}>1 ; w_{1}>1, w_{2}<1 ; w_{1}<1, w_{2}<1$; respectively. We observe that equilibrium point $Q_{4}(0,0)$ always is unstability.

\section{Diagrams of Phase Trajectory}

In order to analysis phase trajectory of the population model (2), we divide the phase plane into several parts by the following straight lines 


$$
L_{1}: 1-\frac{y_{1}}{N_{1}}-w_{1} \frac{y_{2}}{N_{2}}=0, \quad L_{2}: 1-w_{2} \frac{y_{1}}{N_{1}}-\frac{y_{2}}{N_{2}}=0 .
$$

In the case of $w_{1}<1, w_{2}>1$, the phase plane is divided into three parts as shown in Fig 1 . In the case of $w_{1}>1, w_{2}<1$, the phase plane is divided into three parts as shown in Fig 2 . In the case of $w_{1}<1, w_{2}<1$, the phase plane is divided into four parts as shown in Fig 3. In the case of $w_{1}>1, w_{2}>1$, the phase plane is divided into four parts as shown in Fig 4. Taking initial value in each part of phase plane, we write matlab programs for the model (2), and draw the corresponding diagrams of phase trajectory.

In the case of $w_{1}<1, w_{2}>1$, let $w_{1}=0.5, w_{2}=1.6, m_{1}=2.5, m_{2}=1.8, N_{1}=1.6, N_{2}=1$, we write the following matlab programs(see [2]).

The function file :

function $\mathrm{dy}=\mathrm{f} 1(\mathrm{t}, \mathrm{y})$

$\mathrm{dy}=\left[\left(2.5^{*} \mathrm{y}(1)\right) \cdot *(1-\mathrm{y}(1) / 1.6-0.5 * \mathrm{y}(2)),\left(1.8^{*} \mathrm{y}(2)\right) *(1-\mathrm{y}(1)-\mathrm{y}(2))\right]^{\prime}$;

The command file

[t,y]=ode45('f1',[0 5],[0.1 0.1]);

$\operatorname{plot}(\mathrm{y}(:, 1), \mathrm{y}(:, 2))$;

$\%$ Taking initial value $\mathrm{y} 1(0)=0.1, \mathrm{y} 2(0)=0.1$ in $\mathrm{S} 1$

xlabel('y1 axis ');ylabel('y2 axis ');

$\%$ drawing the corresponding diagrams of phase trajectory

hold on

$\operatorname{axis}([0,2,0,2])$;

grid on

$\operatorname{plot}\left(\mathrm{y}, 2-\left(5^{*} \mathrm{y}\right) / 4\right)$;

$\%$ drawing the line $\mathrm{L} 1$

hold on

$\operatorname{plot}\left(\mathrm{y},-\mathrm{y}+1, \mathrm{C}^{-}\right)$; $\quad$ \% drawing the line L2

gtext('y2=-y1+1');

gtext('y2=-(5*y1)/4+2');

title('the population trajectory graph ')

hold on

[t,y]=ode45('f1',[0 5],[1 1]); $\quad \%$ Taking initial value $y 1(0)=1, y 2(0)=1$ in S3

$\operatorname{plot}(\mathrm{y}(:, 1), \mathrm{y}(:, 2))$;

$\%$ drawing the corresponding diagrams of phase trajectory

hold on

[t,y]=ode45('f1',[0 5],[1.8 1.6]); \% Taking initial value y1(0)=1.8,y2(0)=1.6 in S3

$\operatorname{plot}(\mathrm{y}(:, 1), \mathrm{y}(:, 2))$;

$\%$ drawing the corresponding diagrams of phase trajectory

hold on

$[\mathrm{t}, \mathrm{y}]=\mathrm{ode} 45(\mathrm{f} 1 \mathrm{1},[0 \mathrm{0}$ 5],[0.6 1]); \% Taking initial value $\mathrm{y} 1(0)=0.6, \mathrm{y} 2(0)=1$ in $\mathrm{S} 2$

$\operatorname{plot}(\mathrm{y}(:, 1), \mathrm{y}(:, 2))$

$\%$ drawing the corresponding diagrams of phase trajectory

Running the command file, we obtain Fig. 1.

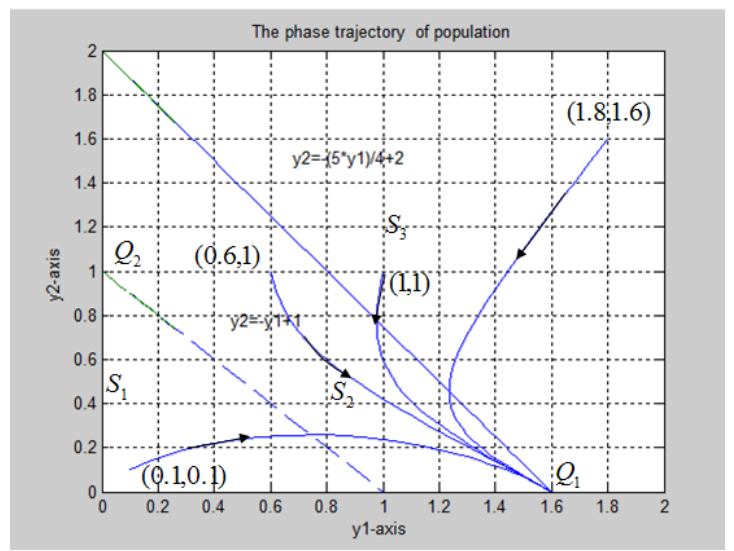

Figure 1. Diagrams of phase trajectory

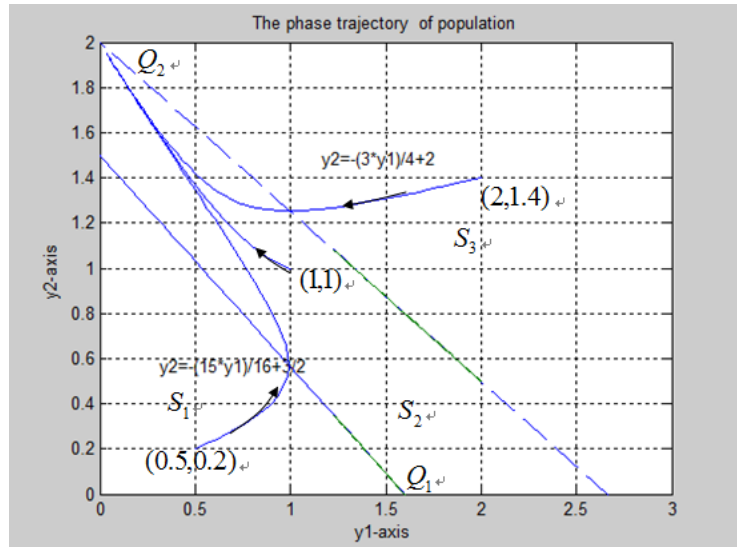

Figure 2. Diagrams of phase trajectory 
In the case of $w_{1}>1, w_{2}<1$, let $w_{1}=4 / 3, w_{2}=0.6, m_{1}=2.5, m_{2}=1.8, N_{1}=1, N_{2}=1.4$, we write matlab programs similar to the matlab programs for the case of $w_{1}<1, w_{2}>1$. Running the command file, we obtain Fig. 2. In the case of $w_{1}<1, w_{2}<1$, let $w_{1}=5 / 7, w_{2}=0.6, m_{1}=2.5, m_{2}=1.8$, $N_{1}=1, N_{2}=1$, we write matlab programs similar to the matlab programs for the case of $w_{1}<1, w_{2}>1$. Running the command file, we obtain Fig. 3. In the case of $w_{1}>1, w_{2}>1$, let $w_{1}=4 / 3, w_{2}=5 / 3, m_{1}=2.5, m_{2}=1.8, N_{1}=2, N_{2}=1.6$, we write matlab programs similar to the matlab programs for the case of $w_{1}<1, w_{2}>1$. Running the command file, we obtain Fig. 4.

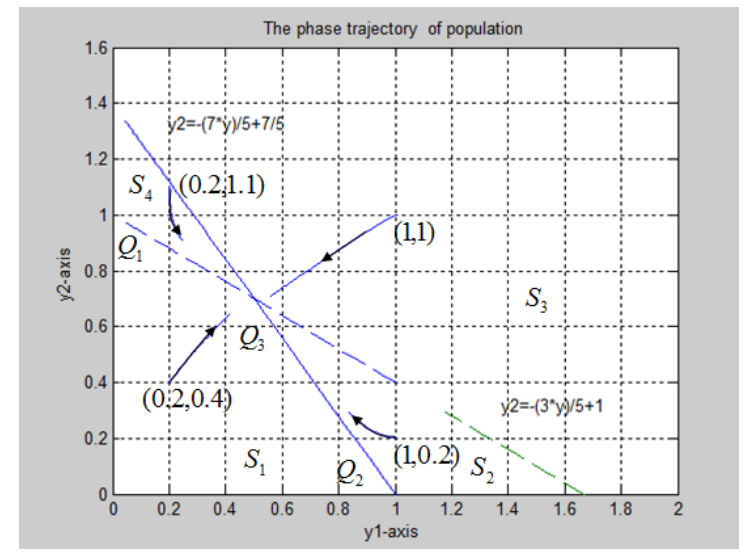

Figure 3. Diagrams of phase trajectory

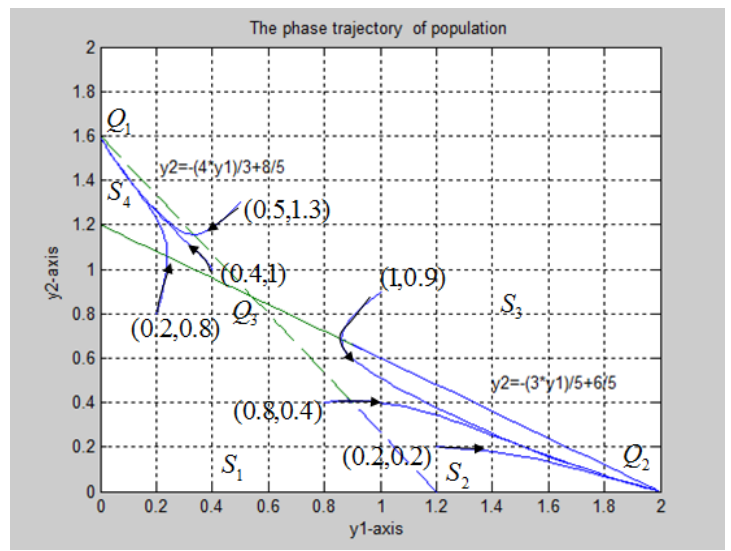

Figure 4. Diagrams of phase trajectory

\section{Summary}

From Fig. 1, we observe that in the case of $w_{1}<1, w_{2}>1$, the all phase trajectory tend to the equilibrium point $Q_{1}\left(N_{1}, 0\right)$. From Fig. 2, we observe that in the case of $w_{1}>1, w_{2}<1$, all phase trajectory tend to the equilibrium point $Q_{2}\left(0, N_{2}\right)$. From Fig. 3, we observe that in the case of $w_{1}<1, w_{2}<1$, all phase trajectory tend to the equilibrium point $Q_{3}\left(\frac{N_{1}\left(1-w_{1}\right)}{1-w_{1} w_{2}}, \frac{N_{2}\left(1-w_{2}\right)}{1-w_{1} w_{2}}\right)$. From Fig 4 , we observe that in the case of $w_{1}>1, w_{2}>1$, the straight lines $L_{1}$ and $L_{2}$ divide the phase plan into four parts $S_{1}, S_{2}, S_{3}$ and $S_{4}$, the all phase trajectory get to $S_{2}$ or $S_{4}$, all phase trajectory which get to $S_{2}$ tend to the equilibrium point $Q_{2}\left(0, N_{2}\right)$, and all phase trajectory which get to $S_{4}$ tend to the equilibrium point $Q_{1}\left(N_{1}, 0\right)$. you follow the "checklist" your paper will conform to the requirements of the publisher and facilitate a problem-free publication process.

\section{Acknowledgements}

This research was supported by National Natural Science Foundation of China (Project No. 11161018, 11561019) and Scientific Research Foundation of the Education Department of Guangxi Autonomous Region of China (No. 201106LX599).

\section{References}

[1] Q. Jiang, J. Xie and J. Ye, Mathematical Model, third ed, Higher Education Press, China, 2003, p.184.

[2] M. Han, J. Wang and L. Li, Mathematics Experiment (Matlab Version), Tongji University Press, China, 2012, p.64. 
[3] W. Xu, Statistical Analysis of Ecological Mathematical Model Based on Differential Equation, Ph.D., Northeast Forestry University, China 2009), p.9.

[4] F. Lu and Z. Li, Interspecific competition model and application of plants in sandy area, Acta Botanica Boreali-Occidentalia Sinica, Vol. 23(2003), No. 1,138-140.

[5] L. Han, Z. Ma and T. Shi, An autonomous SIS epidemic model with standard incidence of two competitive species, Journal of Engineering Mathematics, Vol. 20 (2003), No. 4, 70-74.

[6] B. Hai, The competitive coexistence model of sand-fixation plants, Journal of Northwest University for Nationalities (Natural Science), Vol. 29 (2008), No. 72, 17-24.

[7] Z. Zhao, L. Chen and X. Song, Impulsive vaccination of SEIR epidemic model with time delay and nonlinear incidence rate, Mathematical and Computer in Simulation, Vol. 79 (2008), No. 3, 500-510.

[8] H.F. Huo and Z.P. Ma, Dynamics of a delayed epidemic model with non-monotonic incidence rate, Communications in Nonlinear Science and Numerical Simulation, Vol.15 (2010) 459-468.

[9] X. B. Zhang, H. F. Huo, H. Xiang, et al, An SIRS epidemic model with pulse vaccination and non-monotonic incidence rate, Nonlinear Analysis: Hybrid Systems, Vol. 8 (2013) 13-21.

[10] W. Zhou and G. Hou, SEIR epidemic model of two competitive Species with pulse vaccination, Journal of Anqing Teachers College( Natural Science Edition), Vol. 21 (2015), No. 4, 7-11. (In Chinese) 